\title{
Erratum to: Generation of Electric and Magnetic Fields during High-Intensity Laser Radiation Propagation through the Atmosphere
}

\author{
V. F. Myshkin ${ }^{a}$, S. F. Balandin ${ }^{b}$, V. A. Donchenko ${ }^{c, *}$, V. A. Pogodaev ${ }^{b}$, V. A. Khan ${ }^{a, b, * *}$, E. S. Abramova ${ }^{d}$, \\ Yu. I. Kulakov ${ }^{c}$, M. S. Pavlova ${ }^{d}$, V. L. Khazan ${ }^{e}$, and D. M. Horohorin ${ }^{a}$ \\ ${ }^{a}$ National Research Tomsk Polytechnic University, Tomsk, 634050 Russia \\ ${ }^{b}$ V.E. Zuev Institute of Atmospheric Optics, Siberian Branch, Russian Academy of Sciences, Tomsk, 634055 Russia \\ ${ }^{c}$ Siberian Physical-Technical Institute, Tomsk State University, Tomsk, 634050 Russia \\ ${ }^{d}$ Siberian State University of Telecommunications and Information Sciences, Novosibirsk, 630009 Russia \\ ${ }^{e}$ Omsk State Technical University, Omsk, 644050 Russia \\ *e-mail: gos100@tpu.ru \\ **e-mail: nt.centre@mail.ru \\ Submitted December 11, 2020; accepted for publication December 11, 2020
}

DOI: $10.1134 / \mathrm{S} 1024856020060263$

The affiliation of V. F. Myshkin, V. A. Khan, and D. M. Horohorin under indication $a$ should read as follows:

National Research Tomsk Polytechnic University

The original article can be found online at https://doi.org/10.1134/S1024856020050139 